\title{
What Role Do Intelligent Reflecting Surfaces Play in Non-Orthogonal Multiple Access?
}

\author{
Arthur S. de Sena, Student Member, IEEE, Dick Carrillo, Student Member, IEEE, Fang Fang, Member, IEEE, \\ Pedro H. J. Nardelli, Senior Member, IEEE, Daniel B. da Costa, Senior Member, IEEE, Ugo S. Dias, Senior \\ Member, IEEE, Zhiguo Ding, Fellow, IEEE, Constantinos B. Papadias, Fellow, IEEE, \\ and Walid Saad, Fellow, IEEE
}

\begin{abstract}
Massive multiple-input multiple-output (MIMO) and non-orthogonal multiple access (NOMA) are two key techniques for enabling massive connectivity in future wireless networks. A massive MIMO-NOMA system can deliver remarkable spectral improvements and reduced communication latency. Nevertheless, the uncontrollable stochastic behavior of the wireless channels can still degrade its performance. In this context, intelligent reflecting surface (IRS) has arisen as a promising technology for smartly overcoming the harmful effects of the wireless environment. The disruptive IRS concept of controlling the propagation channels via software can provide attractive performance gains to the communication networks, including higher data rates, improved user fairness, and, possibly, higher energy efficiency. In this article, in contrast to the existing literature, we demonstrate the main roles of IRSs in MIMO-NOMA systems. Specifically, we identify and perform a comprehensive discussion of the main performance gains that can be achieved in IRS-assisted massive MIMO-NOMA (IRS-NOMA) networks. We outline exciting futuristic use case scenarios for IRS-NOMA and expose the main related challenges and future research directions. Furthermore, throughout the article, we support our in-depth discussions with representative numerical results.
\end{abstract}

\section{INTRODUCTION}

The fifth generation of wireless communications $(5 \mathrm{G})$ is already a reality. The first commercial systems were deployed in 2019, and they will continue to be implemented worldwide in the upcoming years [1]. 5G is believed to enable the deployment of demanding applications such as autonomous cars, massive sensor networks, telemedicine, smart homes, and more. To make these applications possible, stringent requirements such as massive connectivity, improved spectrum efficiency, and low communication latency must be fulfilled. Massive multiple-input multiple-output (MIMO) is one of 5G's key technologies for accomplishing these requirements. By exploiting the spatial domain with transmit beamforming techniques, and employing a large number of antennas, massive

A. S. de Sena, Dick C. Melgarejo, and P. H. J. Nardelli are with the Lappeenranta-Lahti University of Technology, Finland.

F. Fang, and Z. Ding are with the University of Manchester, UK.

D. B. da Costa is with the Federal University of Ceará, Brazil.

U. S. Dias is with the University of Brasília, Brazil.

C. B. Papadias is with the Athens Information Technology, Greece.

Walid Saad is with Virginia Tech, USA.

(c) 2020 IEEE. Personal use of this material is permitted. Permission from IEEE must be obtained for all other uses, in any current or future media, including reprinting/republishing this material for advertising or promotional purposes, creating new collective works, for resale or redistribution to servers or lists, or reuse of any copyrighted component of this work in other works.
MIMO schemes enable resource-efficient parallel transmissions to multiple users using the same frequency and time-slot.

Non-orthogonal multiple access (NOMA) is another important technology envisioned to be part of future wireless systems. In particular, by employing superposition coding (SC) at the base station (BS) and successive interference cancellation (SIC) at the receivers, power-domain NOMA can simultaneously serve more than one user within a single resource block. This makes NOMA capable of providing significant connectivity improvements to the communication networks. If massive MIMO and NOMA are properly combined, the features of the two techniques can be exploited to reach even higher spectral gains, which can outperform conventional systems employing orthogonal multiple access (OMA) [2].

Nevertheless, despite their potential improvements, a MIMO-NOMA system has several limitations. The random fluctuation of wireless channels, signal path loss, blocking, high user mobility, and atmospheric absorption are just a few examples of issues that can strongly impact the performance of MIMO-NOMA systems [3]. The impact of such impairments becomes even more severe at higher frequencies, i.e., frequencies above $6 \mathrm{GHz}$, which are a key feature of 5G systems and beyond. Although an increase in the number of antennas can help to overcome such kinds of performance degradation, this comes at the cost of an increased energy consumption when the number of antenna elements becomes high. In view of this, while 5G is still being matured and deployed, engineers and researches have already started looking at new energy-efficient technologies to go beyond $5 \mathrm{G}$ and build the sixth generation (6G) [1]. In particular, due to recent advances in the field of electromagnetic metamaterials, the appealing concept of an intelligent reflecting surface (IRS) has been drawing significant attention from both academia and industry [4].

An IRS is an ultra-thin planar structure composed of a large number of reflecting elements with a size smaller than the signal wavelength, known as meta-atoms [5], [6]. The key advantage of IRS structures is that each meta-atom can be dynamically tuned by software with distinct phases and amplitudes of reflection so that they can collaboratively forward the impinging waves with, ideally, any desired radiation pattern, like a hologram. Such a capability enables the deployment of smart wireless environments with optimized and possibly energy-efficient signal propagation, thus paving the way towards a new wireless communication paradigm. For instance, 


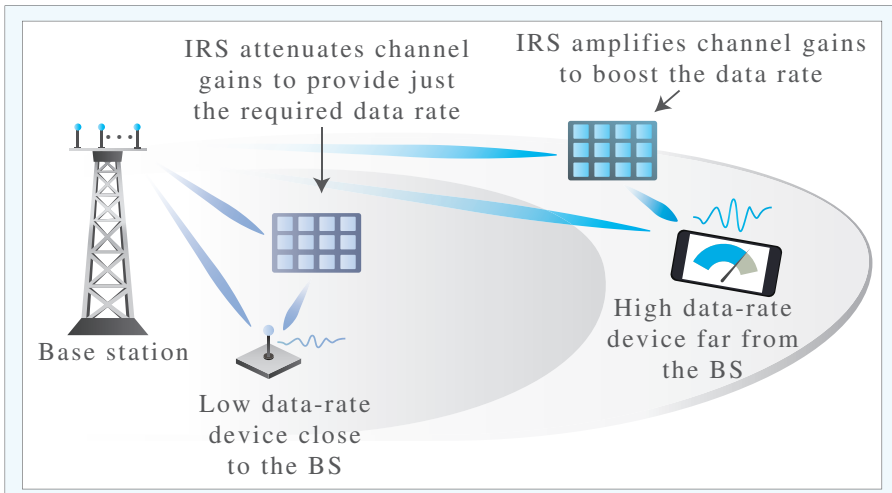

(a)

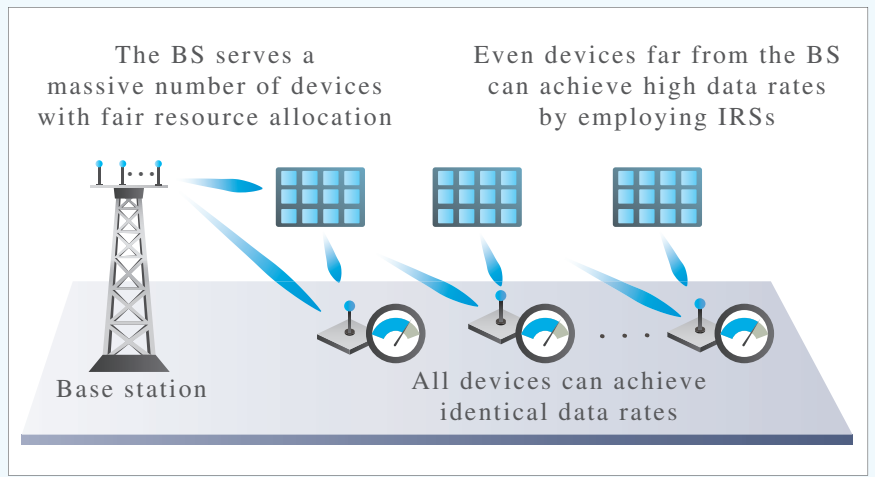

(b)

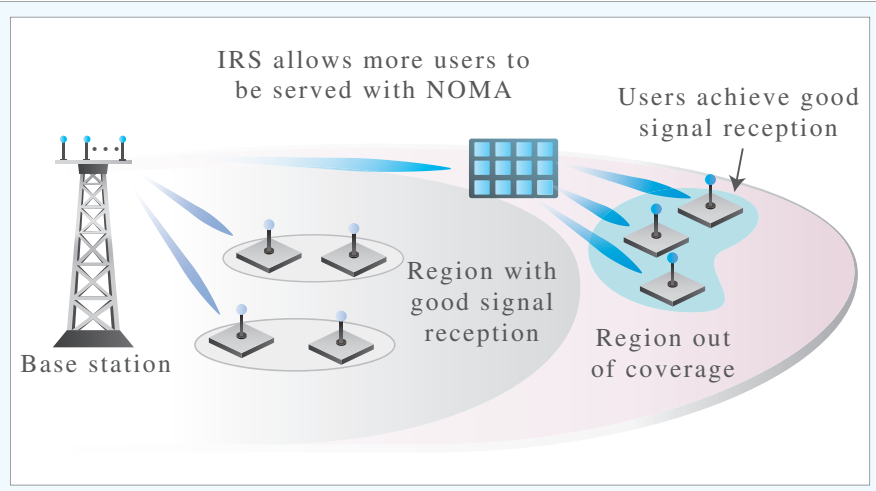

(c)

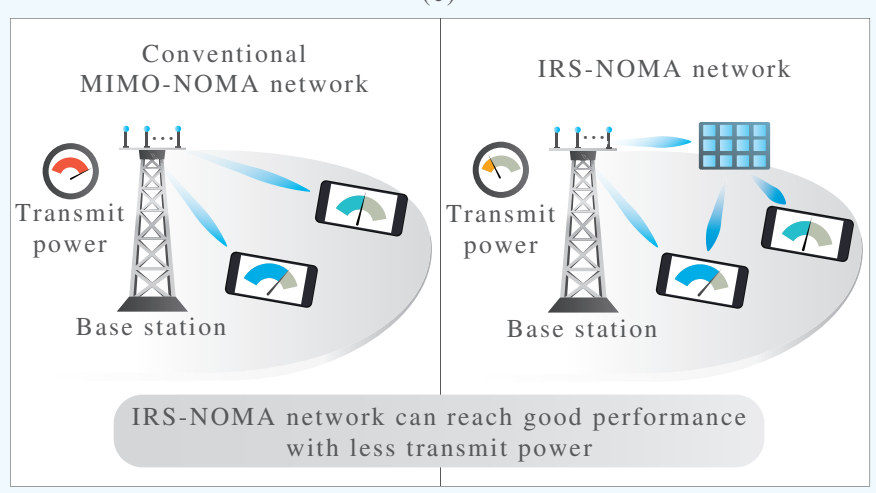

(d)

Fig. 1. Illustration of potential achievements of IRS-NOMA networks. (a) The IRSs can tune the channel gains to meet the data rate requirements of both near and far users. (b) The IRSs can boost the performance of the network under fair resource allocation. (c) By deploying an IRS at the cell-edge, more users can be served with NOMA. (d) The IRS-NOMA system can achieve similar rate performance of conventional MIMO-NOMA with less transmit power.

by properly optimizing the IRS's reflection coefficients, signal beams can be formed to achieve goals such as to enhance the performance of a specific terminal, to cancel interference coming from other devices, or even to completely null out information leakage at an eavesdropper [7], [8]. In addition to these advantageous features, an IRS has potential to exhibit near-zero energy consumption since it does not comprise the power-hungry components of conventional radiofrequency chains. In general, an IRS design contains passive metamaterial parts and an active control components with only ultralow-power electronic circuitry that can be powered by energy harvesting wireless modules [4]. These make IRSs a promising plug-and-play technology for improving the performance of future communication networks.

Although a number of surveys on the topic of IRS have recently appeared in [4], [7], and [9], none of those prior works have overviewed and studied the application of IRSs to MIMO-NOMA networks. As a result, it is still not completely clear what are the roles that IRSs can play in such networks. Therefore, further studies for the in-depth understanding of the combination of these two promising subjects are required. In view of this, the main contribution of this article is to investigate the potential spectral and energy efficiency gains of IRS-assisted massive MIMO-NOMA (IRS-NOMA) systems in future wireless networks as presented in Fig. 1. We will also discuss the fundamental challenges pertaining to their effective deployment. In summary, this article will have the following key contributions:

- To provide general readers with a basic understanding of the IRS technology, a typical IRS architecture and its basic working principle is presented. A commonly adopted channel model is provided, and the main features that differentiate IRS from other related technologies are also highlighted.

- Four attractive potential achievements of IRS-NOMA networks are identified. Specifically, we show that IRSNOMA can enable flexible control on the users' channel gains, enhanced user fairness, enhanced scalability, and improved energy efficiency.

- Pervasive coverage via multiple IRSs, 3D coverage in UAVs networks, and massive grant-free transmissions are introduced as three promising applications that IRSNOMA has potential to enable in future wireless systems beyond $5 \mathrm{G}$.

- Finally, a comprehensive discussion of the main open problems, technical challenges, and possible future directions of IRS-NOMA is provided.

\section{AN OVERVIEW ON THE IRS TECHNOLOGY}

To shed light on the operation of an IRS system, in this section, we provide a fundamental background on IRS hardware architecture, channel model, and a comparison with two different related technologies. 
TABLE I

COMPARISON OF IRS WITH OTHER TECHNOLOGIES

\begin{tabular}{|c|c|c|c|}
\hline Technology & Operation mode & Characters & Drawbacks \\
\hline IRS & Full duplex & $\begin{array}{c}\text { Low hardware cost } \\
\text { Potential to exhibit low energy consumption }\end{array}$ & $\begin{array}{c}\text { Short range of implementation } \\
\text { Difficult to estimate CSI }\end{array}$ \\
\hline AF relay & Half/full duplex & Actively regenerate and transmit signals & $\begin{array}{c}\text { High hardware cost } \\
\text { High energy consumption }\end{array}$ \\
\hline Ambient Backscatter & Half duplex & $\begin{array}{c}\text { Low hardware cost } \\
\text { Low energy consumption }\end{array}$ & $\begin{array}{c}\text { Limited data rate } \\
\text { Strong interference from active source }\end{array}$ \\
\hline
\end{tabular}

\section{A. Fundamentals of IRS's Architecture}

The IRS architecture is not a unified subject. The available literature proposes a variety of IRS designs with a different number of layers and different technologies, including liquid crystals, microelectromechanical systems, doped semiconductors, and electromechanical switches [4]. Despite that, the majority of the architectures share at least three common layers, which consist of (1) a meta-atom layer, comprising a larger number of passive conductor elements and low power active switches; (2) a control layer, which is responsible for adjusting the amplitude and phase shift of each meta-atom element; and (3) a gateway layer (or communication layer) that establishes the communication between the control layer and the BS. Each meta-atom acts as a sub-wavelength scatterer with reconfigurable electromagnetic properties. Such a feature enables them to collectively change the induced current patterns in the IRS so that a desired electromagnetic field response can be generated. This allows the IRS to manipulate the wavefronts to achieve objectives like steering, absorption, polarization, filtering, and collimation [9].

\section{B. IRS's Channel Model}

In a general IRS-aided MIMO transmission model, the channel matrix from the BS to the user, via an IRS, includes the channel responses from BS to the IRS, a diagonal matrix that models the IRS's signal reflection, and the channel responses between the IRS and the user. Specifically, the IRS receives the signal from the BS, and then reflect the incident signal by inducing the amplitude and phase changes adjusted by the control layer. As a result, the BS-IRS-user link can be represented by a multiplicative channel model, which can be added coherently with the direct link from the BS to either boost or attenuate the signal strength at the receiver [7].

\section{IRS and Related Technologies}

Next, we discuss some important features that make IRSs distinct from related technologies like amplify-and-forward (AF) relaying and ambient backscatter radio systems.

In AF relaying networks, when a relay node amplifies the received signals (which can be energy-consuming), it also amplifies noise, which consequently can degrade the system performance. Moreover, AF relays can only operate in full-duplex mode if efficient self-interference cancellation techniques are employed. This impairment can increase the implementation cost and system complexity [7]. In contrast, the IRSs operate in passive reflecting mode and do not require a dedicated energy source to retransmit the impinging signals. Such characteristic enables the IRSs to work in a full-duplex mode without generating self-interference and noise amplification. Ambient backscattering communication [10] is another technology that operates recycling the impinging electromagnetic waves. However, the working principle and objectives of such systems are very distinct from the IRS concept. While IRSs are designed only to reflect transmitted signals, the passive backscattering devices harvest energy from the received analog waves coming from different active sources to transmit its own information. Furthermore, backscatter systems are susceptible to strong direct interference generated from the active sources, an issue that is not present in IRS networks. A comparison of the features of IRS and the aforementioned technologies is summarized in Table I.

\section{IRS-NOMA Networks: POTENTIAL IMPROVEMENTS}

In this section, we raise four important performance gains that IRS-NOMA systems can potentially provide, namely tuned channel gains, improved fair resource allocation, enhanced coverage range, and high energy efficiency. These attractive achievements are illustrated in Fig. 1. For each of the illustrated gains, we perform a comprehensive and in-depth discussion that is supported by numerical results generated from IRS-NOMA Monte Carlo simulations. In particular, we consider the downlink transmissions of a cellular network having a single BS equipped with a uniform linear array of 80 transmit antennas that serves users with 4 receive antennas. Unless stated otherwise, an IRS with 20 reflecting elements is installed nearby to each user, where it is assumed that the IRS of a given user does not interfere with other IRSs. Furthermore, we present results considering both fixed and optimized reflection coefficients. In the optimized IRS results, depending on the system objectives, we dynamically tune the reflecting elements to either maximize or attenuate the instantaneous rates achieved by each user, and, on the fixed setups, all reflection phases and amplitudes are adjusted to $0^{\circ}$ and 1 , respectively. Moreover, analogously to [2] and [3], the multi-antenna users are considered to be distributed among multiple clusters, in which the users are sub-divided into multiple NOMA groups. In order to cancel inter-cluster interference, the BS employs a precoder that is constructed based on the null-space spanned by the channel matrices 


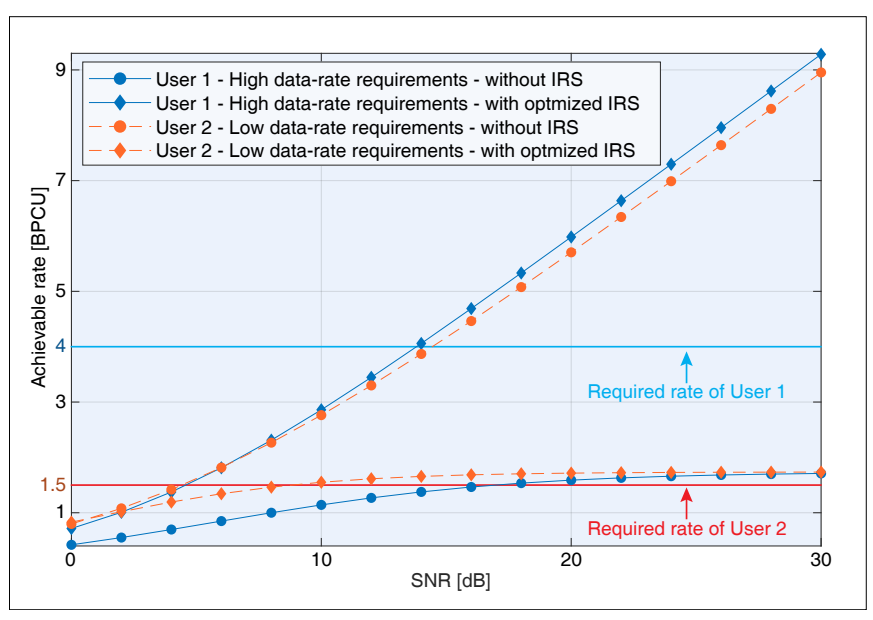

Fig. 2. Achievable rates versus SNR for MIMO-NOMA and IRS-NOMA systems under fixed power allocation where user 1 is located at $200 \mathrm{~m}$ and user 2 at $100 \mathrm{~m}$ from the BS. The power allocation coefficients of users 1 and 2 are $7 / 10$ and $3 / 10$, respectively.

of interfering clusters, and each user adopts a zero-forcing receiver to eliminate the remaining inter-group interference. More details about this transmission and reception strategy can be found in [2].

\section{A. Tuned Channel Gains}

In order for NOMA to be effective, during the SC process at the BS, the users are sorted out in ascending or descending order based on their channel gains so that, relying on this information, the users can successfully employ SIC to recover the transmitted messages. This renders the performance of MIMO-NOMA systems highly dependent on the users' channel conditions. Specifically, it has been shown that NOMA can achieve higher spectral efficiency than OMA only if the channel gains of the different users are significantly distinct and if their spatial directions are not orthogonal to each other [11], [12]. The challenge of these constraints in conventional networks is that they can not always be satisfied. This is because the highly stochastic propagation paths are determined almost exclusively by the scattering environment and the location of the receivers, in which classical communication systems have no control. This scenario completely changes when it comes to IRS-assisted networks. By deploying IRSs, the propagation environment can be smartly tuned according to the desired objectives, potentially enabling the network to finely adjust its users' channel gains so that NOMA can always achieve good spectral efficiency. For instance, the recent work in [12] has shown that, with the help of IRSs, it is possible to force the channels to become quasi-degraded, which is a condition in which MIMO-NOMA can achieve the same performance of dirty paper coding (DPC), i.e., where it can approach the capacity region of the downlink channel.

The IRS technology introduces a new paradigm to MIMONOMA networks by providing it with flexibility in multiplexing users. By employing IRSs, it can become possible even to change the original order of the users' channel gains. This capability enables the network to sort its users based on their particular data rate requirements rather than on the uncontrollable random environment of classical communication systems. For instance, in conventional NOMA deployments with fixed power allocation, when a user with high capacity requirements faces highly unfavorable channel conditions, e.g., when the user is very far from the BS, it will inevitably fall in an outage state (a state where its minimum performance requirements are not met). This is a difficult situation to be solved by traditional approaches since, independently of the power allocated, the weak user's rate will always be limited due to interference from the strong user. Fig. 2 shows the simulation results for the scenario illustrated in Fig. 1a, where user 1 is located at $200 \mathrm{~m}$ and user 2 at 100 from the BS. We see that the conventional MIMO-NOMA system is not able to deliver the required rate of 4 bits per channel use (BPCU) for user 1 . On the other hand, user 2 , with a low data rate requirement, is reaching a channel capacity much higher than necessary. By employing IRSs in this scenario, the system performance can be efficiently optimized. As one can notice, by properly adjusting the reflection coefficients of the IRSs, user 1 that was originally experiencing bad channel conditions can achieve high performance and meet its required data rate. In contrast, the channel gains of the nearer user 2 are optimized to provide just the necessary capacity.

\section{B. Improved Fair Resource Allocation}

In some emerging applications, such as the Industrial Internet of Things, it can be important that all devices experience similar data rates. It has been demonstrated that, by properly performing power allocation, MIMO-NOMA systems can achieve this interesting capability. Specifically, the performance of different devices can be balanced by maximizing the minimum achievable rate in the network so that everyone can experience similar rate levels. The main disadvantage of such approaches is that to increase the performance of weak device, the ones with good channel conditions can be excessively penalized. In addition, if the channel conditions of the weak device are too degraded, the data rates achieved under fair power allocation can be not enough to meet the quality of service requirements of other devices, leading to poor network performance. As illustrated in Fig. 1b, installing IRSs in such deployments can be very beneficial. Theoretically, all devices could reach the same data rate with the help of dynamic fair power allocation, while the IRSs could boost the channel gains to guarantee a high network performance.

This above achievement can be visualized in the simulation results presented in Fig. 3, where we employ to MIMONOMA and IRS-NOMA both fixed and the fair power allocation policy developed in [13]. Here, we consider the existence of two users per NOMA group, one located at $100 \mathrm{~m}$ and another at $200 \mathrm{~m}$ from the BS. One can see that the fair power allocation in the MIMO-NOMA system can successfully balance the users' rates. However, the strong user pays an expensive price for enabling this capability. For instance, when the signal-to-noise ratio (SNR) is $36 \mathrm{~dB}$, the rate achieved by both users in MIMO-NOMA under fair power allocation is $4 \mathrm{BPCU}$. This represents an expressive reduction 


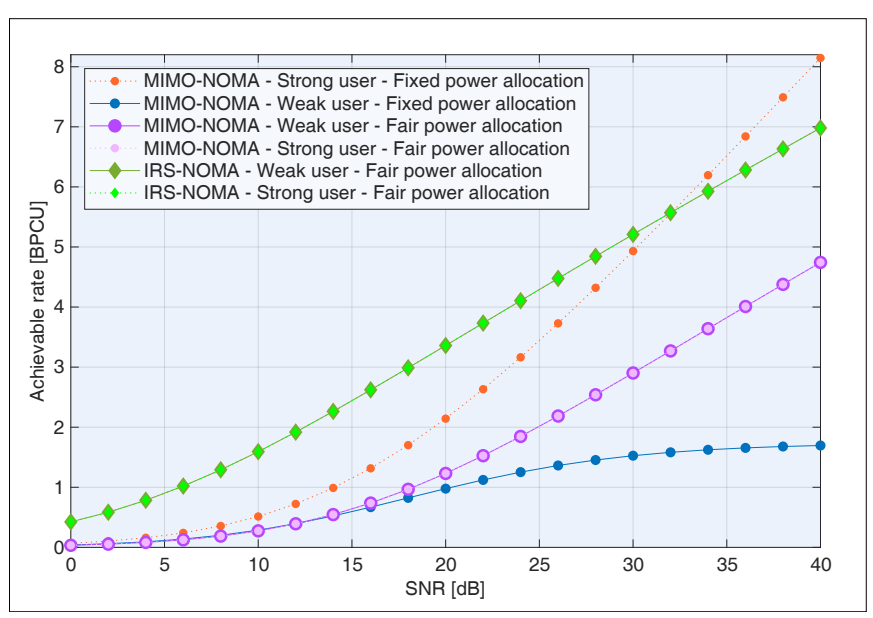

Fig. 3. Achievable rates versus SNR for MIMO-NOMA and IRS-NOMA systems under fixed and fair power allocation, where the weak user is located at $200 \mathrm{~m}$ and the strong one at $100 \mathrm{~m}$ from the BS. When fixed allocation is employed, the power coefficients for the weak and strong users are $7 / 10$ and $3 / 10$, respectively.

of almost $3 \mathrm{BPCU}$ to the strong user's rate. On the other hand, the IRS-NOMA, in which we consider fixed reflection coefficients, can provide a high data rate performance for all users. The rate curves become superior even to that achieved by the strong one with fixed allocation, for the majority of the considered SNR range. This result confirms that IRS-NOMA can bring high-performance fair networks to reality in the future.

\section{Enhanced Coverage Range}

In conventional wireless networks, it is difficult to provide uniform signal coverage to all existing devices. Users that are far from a BS, or that are suffering heavy blockage may experience poor or no signal reception. This issue becomes more relevant at the higher frequency bands of $5 \mathrm{G}$, $6 \mathrm{G}$, and beyond. The short wavelengths of the millimeterwave and terahertz spectrum can resonate with atmospheric oxygen and water molecules, making a significant part of the radiated energy to be dissipated through kinetic absorption. This harmful characteristic can cause strong signal attenuation, leading to a substantial decrease in the communication range. One conventional way for overcoming this short coverage impairment can be achieved with a dense deployment of BSs. However, such approach would result in high infrastructure costs. Fortunately, IRS-NOMA systems are capable of enabling long-range communication with low deployment costs. As shown in Fig. 1c, an IRS could be installed at the celledge to enhance the transmission range and cover users that are located in a region with no signal reception. As a result, more users can be served with NOMA, thereby enhancing the connectivity capacity of the system.

A practical demonstration of the aforementioned IRS capability can be seen in Fig. 4. This simulation example shows the achievable rates for MIMO-NOMA and IRS-NOMA networks under fixed power allocation when the SNR is $26 \mathrm{dBm}$. More specifically, we consider three users in the group of interest. Two users are closer to the BS, with the nearest

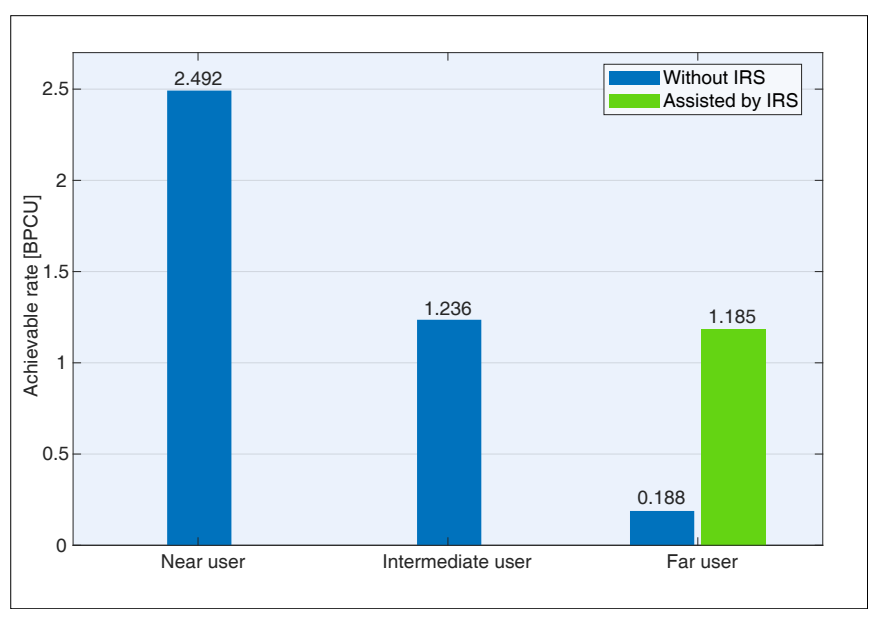

Fig. 4. Achievable rates when the SNR is $26 \mathrm{dBm}$ for MIMO-NOMA and IRS-NOMA systems serving three users with fixed power allocation. The near user is located at $100 \mathrm{~m}$, the intermediate user at $200 \mathrm{~m}$, and the far user at $1500 \mathrm{~m}$ from the BS. The power coefficients for the near, intermediate, and far users are $1 / 10,3 / 10$ and $6 / 10$, respectively.

user located at $100 \mathrm{~m}$ and the intermediate one at $200 \mathrm{~m}$, while the third user is very far from the BS, located at $1500 \mathrm{~m}$. As one can observe, in the conventional MIMONOMA system, due to the long distance from the BS, the far user is experiencing a weak signal reception that allows a data rate of only 0.188 BPCU. When the same user is assisted by an IRS, its rate is improved to $1.185 \mathrm{BPCU}$, which represents a gain of more than 6 times when compared with that achieved in MIMO-NOMA and incredibly almost the same rate obtained by the intermediate user. This result clearly demonstrates another attractive improvement that can be achieved with IRS-NOMA systems.

\section{High Energy Efficiency}

Achieving the benefits discussed in the previous subsections without increasing energy consumption is another potential advantage of IRS-assisted networks. Specifically, since the meta-atoms of an IRS are passive elements, the only energy consumption needed to enable the IRS's reconfigurability capability, which can be implemented with the help of ultralow-power electronics. In consequence, the use of energy harvesting components can be enough to supply all the necessary power, providing the IRS technology with an opportunity to become truly energy-neutral. As shown in Fig 1d, if this attractive feature becomes a reality, we can achieve higher performance gains with less transmit power, significantly improving the energy efficiency of the network. Nevertheless, it is noteworthy that, if the energy neutrality assumption can not be satisfied, the energy efficiency will inevitably be decreased.

In Fig. 5, we present the energy efficiency curves versus transmit power for MIMO-NOMA and IRS-NOMA schemes. In order to show how energy efficient IRSs can become, we consider the scenario in which energy neutrality can be achieved, and as well as scenarios where the IRS contributes to the power consumption of the network. As one can see, when energy neutrality is considered, the IRS-NOMA system can offer remarkable energy efficiency improvements that 


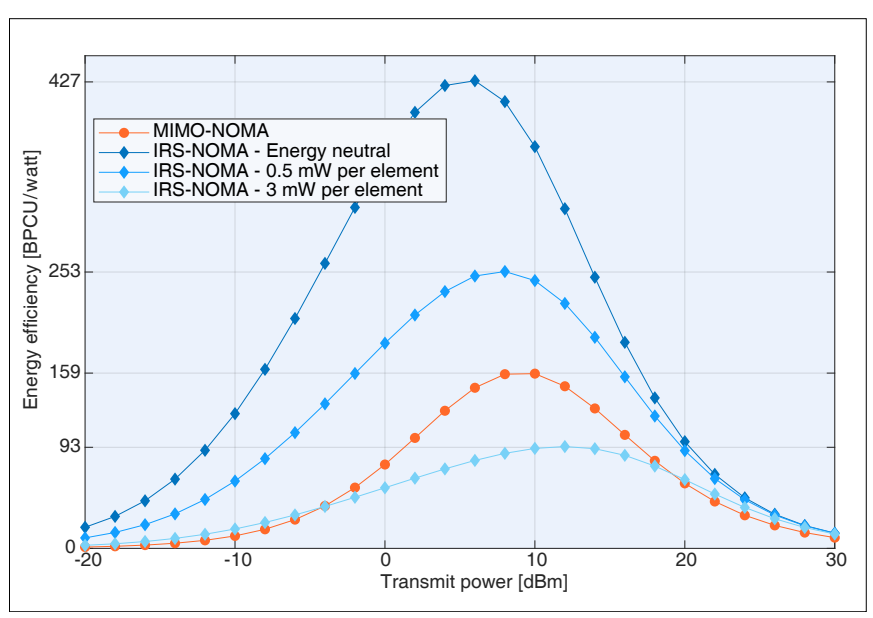

Fig. 5. Energy efficiency versus transmit power for MIMO-NOMA and IRSNOMA systems under fixed power allocation. Two users are considered in this simulation, one located at $100 \mathrm{~m}$ and another at $200 \mathrm{~m}$ from the BS. The power coefficients for the far and near users are $7 / 10$ and $3 / 10$, respectively.

outperform the conventional MIMO-NOMA counterpart. For example, when the transmit power is $10 \mathrm{dBm}$, the conventional MIMO-NOMA system can reach a maximum energy efficiency of 159.6 BPCU/Watt, while with the IRS-NOMA scheme, the maximum energy efficiency increases up to incredible $427.2 \mathrm{BPCU} / \mathrm{Watt}$ and, at the same time, the transmit power required to reach this point decreases to $6 \mathrm{dBm}$. However, when the IRS's energy consumption is taken into account, the energy efficiency is strongly impacted. For instance, if we consider that each reflecting element introduces an additional $0.5 \mathrm{~mW}$ to the total power consumption, the maximum energy efficiency is decreased to approximately $253 \mathrm{BPCU} /$ Watt, and when the power consumption per element is $3 \mathrm{~mW}$, the energy efficiency becomes inferior to that achieved in the conventional MIMO-NOMA system. This behavior demonstrates that IRSNOMA networks are only capable of improving energy efficiency if the power consumed by the IRS is negligible.

\section{SCEnARIOS AND OpPoRTUNities}

In this section, we identify and discuss potential IRSNOMA use case scenarios for future wireless networks.

\section{A. Multiple IRSs for Pervasive Coverage}

The majority of the existing IRS-NOMA related works consider system models where users are assisted by a single IRS. However, as the technology matures and its cost drops down, one can envision a wide-scale deployment of multiple IRSs for pervasive coverage. In such scenarios, the IRSs could be jointly coordinated to deliver multiple independent beams to each user so that they could achieve high channel capacity and ubiquitous signal reception. This large-scale IRS-NOMA network could provide massive access and, simultaneously, enable the deployment of futuristic applications with ultrahigh data rate requirements such as holographic augmented reality and telepresence. For instance, in the future, large-scale IRS-NOMA can be deployed in crowded environments, like shopping malls, to attend the massive number of connections and provide ultra-high throughput to the users' sophisticated holographic enabled smartphones.

\section{B. 3D Coverage in UAV Networks}

The deployment of unmanned aerial vehicles (UAV) as aerial BSs is another appealing approach for improving signal coverage of the communication networks. The intelligent arrangement of multiple UAVs combined with their high operating altitude enables them to enhance the coverage area through efficient dynamic 3D beamforming. UAV networks can offer many advantages, such as flexibility in increasing the number of transmit antennas (with any array pattern), the ability to avoid obstacles (avoiding signal blockage), and more [14]. In addition to that, if NOMA is applied to UAV networks, it becomes possible to serve multiple devices with a single 3D beam, which is attractive for enabling massive access in ultradense dynamic environments.

Despite the above advantages, UAVs face a major impairment that is related to their limited energy supply. Specifically, since each UAV operates as an active BS, the energy required to power on its radiofrequency circuitry can substantially reduce their on-board batteries' lifetime. One could install high capacity batteries to alleviate this issue. However, due to the high cost of such energy storage units, the network can become excessively expensive. Therefore, strategies for reducing energy consumption in UAV networks are crucial for their successful implementation. The employment of IRSs can be very effective for accomplishing this objective as well. For instance, instead of deploying all UAVs as active transmitters, we could implement hybrid networks where part of the UAVs work as active, and others operate as smart reflective devices equipped with only light-weight passive IRSs. This architecture would provide extended signal coverage by exploiting a combination of passive and active 3D beamforming, leading to a reduction in network costs, reduced energy consumption (if IRS energy neutrality is satisfied), and prolonged UAVs' lifetime. For example, a UAV assisted IRS-NOMA network could provide 3D coverage and enable massive access for multiple groups of devices located on different floors of a building.

\section{Massive Grant-free Transmissions}

Grant-free protocols have arisen as efficient approaches for reducing the high signaling overhead faced in traditional cellular networks [15]. In particular, NOMA-based grant-free transmissions allow multiple devices to transmit information in the uplink using the same spectrum, and without requiring grant to radio resources from the BS. Such schemes can efficiently tackle collision issues and reduce communication delays, making them ideal for enabling critical applications with ultra-low latency requirements. However, when the number of connected devices grows, the probability of achieving similar channel gains at the BS also increases. This characteristic can lead to poor multiuser detection performance. For example, in massive machine type communication applications like in a car factory, such impairment could lead to a failure in the production line. The installation of IRSs in this scenario can 
reduce the likelihood of happening this serious issue. IRSNOMA-based grant-free transmissions could guarantee the BS to be able to always distinguish the different devices so that the network can operate with a stable performance even when the number of connections becomes large.

\section{Research Challenges and Future Directions}

Together with the possible improvements and exciting use case scenarios of IRS-NOMA systems, comes also new challenges and open problems.

In Section III, we showed that in IRS-NOMA networks, the order of the users could be efficiently controlled by adequately configuring the IRS's meta-atoms. However, to enable this capability, the IRS must be dynamically optimized based on the instantaneous channel realizations. This imposes major challenges for the practical implementation of IRS-NOMA networks, once that such an optimization can become highly complex as the number of reflecting elements increases. Therefore, the development of efficient low-complexity algorithms for optimizing the IRS coefficients is mandatory and thereby a promising research direction.

User clustering schemes also play an important role in the performance of IRS-NOMA systems. For instance, in [12], it was shown that if users with certain channel conditions are grouped, IRS-NOMA can be outperformed by the IRS-OMA counterpart. This demonstrates that the design of efficient user clustering algorithms is also essential to exploit at maximum the benefits of IRS-NOMA systems. Given that only a few works have investigated this subject until now, this field of research allows for excellent opportunities of future studies. Furthermore, the fact that IRS-OMA can outperform IRSNOMA also raises another issue: when should we use NOMA, and when should we use OMA? Such a fundamental question is not yet totally clarified and deserves further investigation.

It was highlighted that, if energy neutrality is satisfied, an IRS can be able to provide remarkable energy efficiency gains to the communication system. However, to bring this capability to reality, many improvements to the IRS technology need to be achieved. Energy-efficient metamaterials must be engineered, the IRS's embedded circuit components should be optimized to require the lowest possible energy supply, and the optimization process of meta-atoms has to impose low computational complexity to reduce the energy consumed with data processing. Otherwise, the employment of IRSs can lead in the opposite direction; that is, it can increase energy consumption and, consequently, reduce the energy efficiency of the network.

The idea of a large-scale deployment of IRSs, as suggested in Section IV-A, can also bring challenges to the network. The joint coordination of multiple IRSs can trigger an explosion in signaling and processing overhead, which can potentially impact communication latency. In addition, in such scenarios, since the channel gains obtained at the receiver is the result of the combination of all links from all IRSs, achieving the optimal set of reflection coefficients for the entire network can become extremely difficult. Nevertheless, this is an interesting worth studying topic that is still lacking in the literature.
Finally, the concept of hybrid IRS-NOMA UAV networks (with part of the UVSs active and part passive) is also an unexplored subject that can find attractive applications in $6 \mathrm{G}$ and beyond, and, therefore, it is another promising research possibility.

\section{ACKNOWLEDGMENT}

This work is partly supported by Academy of Finland via: (a) ee-IoT project under Grant n.319009, (b) FIREMAN consortium under Grant CHIST-ERA/n.326270, and (c) EnergyNet research fellowship under Grants n.321265 and n.328869. The work is also supported, in part, by the U.S. National Science Foundation (NSF) under Grant 1814477.

\section{REFERENCES}

[1] W. Saad, M. Bennis, and M. Chen, "A Vision of 6G Wireless Systems: Applications, Trends, Technologies, and Open Research Problems," IEEE Network, to appear, 2020.

[2] A. S. de Sena, D. B. da Costa, Z. Ding, and P. H. J. Nardelli, "Massive MIMO-NOMA Networks with Multi-Polarized Antennas," IEEE Trans. Wireless Commun., vol. 18, no. 12, Dec. 2019, pp. 5630-5642.

[3] A. S. de Sena, D. B. da Costa, Z. Ding, P. H. J. Nardelli, U. S. Dias, and C. B. Papadias, "Massive MIMO-NOMA Networks with Successive Sub-Array Activation," IEEE Trans. Wireless Commun., 2019, pp. 1-1.

[4] C. Huang, S. Hu, G. C. Alexandropoulos, A. Zappone, C. Yuen, R. Zhang, M. Di Renzo, and M. Debbah, "Holographic MIMO surfaces for 6G wireless networks: Opportunities, challenges, and trends," [Online] Available: https://arxiv.org/abs/1911.12296, accessed on Jan. 2020.

[5] G. Yang, X. Xu, and Y.-C. Liang, "Intelligent reflecting surface assisted non-orthogonal multiple access," [Online] Available: http://arxiv.org/abs/1907.03133, accessed on Jan. 2020.

[6] I. F. Akyildiz, C. Han, and S. Nie, "Combating the Distance Problem in the Millimeter Wave and Terahertz Frequency Bands," IEEE Commun. Mag., vol. 56, no. 6, Jun. 2018, pp. 102-108.

[7] Q. Wu, and R. Zhang, "Towards Smart and Reconfigurable Environment: Intelligent Reflecting Surface Aided Wireless Network," IEEE Commun. Mag., 2019, pp. 1-8.

[8] M. Jung, W. Saad, M. Bennis, and C. S. Hong, "On the Optimality of Reconfigurable Intelligent Surfaces (RISs): Passive Beamforming, Modulation, and Resource Allocation," [Online] Available: https://arxiv.org/abs/1910.00968, accessed on Jan. 2020.

[9] C. Liaskos, S. Nie, A. Tsioliaridou, A. Pitsillides, S. Ioannidis, and I. Akyildiz, "A New Wireless Communication Paradigm through SoftwareControlled Metasurfaces," IEEE Wireless Commun., vol. 56, no. 9, Sep. 2018, pp. 162-169.

[10] N. V. Huynh, D. T. Hoang, X. Lu, D. Niyato, P. Wang, and D. I. Kim, "Ambient Backscatter Communications: A Contemporary Survey," IEEE Commun. Surv. Tutorials, vol. 20, no. 4, May. 2018, pp. 2889-2922.

[11] M. Vaezi, R. Schober, Z. Ding, and H. V. Poor, "Non-Orthogonal Multiple Access: Common Myths and Critical Questions," IEEE Wireless Commun., vol. 26, no. 5, Oct. 2019, pp. 174-180.

[12] J. Zhu, Y. Huang, J. Wang, K. Navaie, and Z. Ding, "Power Efficient IRS-Assisted NOMA," [Online] Available: https://arxiv.org/abs/1912.11768, accessed on Jan. 2020.

[13] A. S. de Sena, F. R. M. Lima, D. B. da Costa, Z. Ding, P. H. J. Nardelli, U. S. Dias, and C. B. Papadias, "Massive MIMO-NOMA Networks with Imperfect SIC: Design and Fairness Enhancement," [Online] Available: https://doi.org/10.36227/techrxiv.11566725.v1, accessed on Jan. 2020.

[14] M. Mozaffari, W. Saad, M. Bennis, Y. Nam, and M. Debbah, "A Tutorial on UAVs for Wireless Networks: Applications, Challenges, and Open Problems," IEEE Commun. Surv. Tutorials, vol. 21, no. 3, 2019, pp. 2334 $-2360$.

[15] Z. Ding, R. Schober, P. Fan, and H. V. Poor, "Simple Semi-Grant-Free Transmission Strategies Assisted by Non-Orthogonal Multiple Access," IEEE Trans. Commun., vol. 67, no. 6, Jun. 2019, pp. 4464 - 4478. 